\title{
Role of CD11/CD18 in Shear Rate-dependent Leukocyte-Endothelial Cell Interactions in Cat Mesenteric Venules
}

\author{
M. A. Perry and D. N. Granger \\ School of Physiology \& Pharmacology, University of New South Wales, Kensington, 2033 Australia; and Department of Physiology \\ and Biophysics, Louisiana State University Medical Center, Shreveport, Louisiana 71130
}

\begin{abstract}
In vivo microscopy was used to assess the relationships among shear rate (and shear stress), leukocyte rolling velocity, and leukocyte adherence in a cat mesentery preparation. Shear rate in individual venules and arterioles of 25-35 $\mu \mathrm{m}$ diameter were varied over a wide range by graded occlusion of an arterial loop. There was a linear decline in leukocyte rolling velocity $\left(V_{\text {wbc }}\right)$ as red cell velocity $\left(V_{\text {rbc }}\right)$ was reduced. The ratio $V_{\text {wbc }} / V_{\text {rbc }}$ remained constant despite variations in shear stress from 5-25 $\mathrm{dyn} / \mathrm{cm}^{2}$. A reduction in shear stress was associated with an increased leukocyte adherence, particularly when $V_{\text {wbc }}$ was reduced below $50 \mu \mathrm{m} / \mathrm{s}$. Reduction in wall shear rate below 500 $\mathrm{s}^{-1}$ in arterioles allowed 1-3 leukocytes to adhere per $100 \mu \mathrm{m}$ length of vessel, while venules exposed to the same shear rates had 5-16 adherent leukocytes. In arterioles, leukocyte rolling was only observed at low shear rates. At shear rates $<250 \mathrm{~s}^{-1}$ leukocyte rolling velocity was faster in arterioles than venules, and the ratio $V_{\text {wbc }} / V_{\text {rbc }}$ for arterioles was $0.08 \pm 0.02$, which was fourfold higher than the ratio obtained in venules at similar shear rates.

Pretreatment with the CD18-specific antibody (mAb) IB increased leukocyte rolling velocity in venules by $\sim 20 \mu \mathrm{m} / \mathrm{s}$ at red cell velocities below $2,000 \mu \mathrm{m} / \mathrm{s}$. $\mathrm{mAb} \mathrm{IB}_{4}$ largely prevented the leukocyte adherence to arterioles and venules, and increased the ratio $V_{\text {wbc }} / V_{\text {rbc }}$ observed in venules at low shear rates. The results of this study indicate that low shear rates elicit a CD18-dependent adhesive interaction between leukocytes and microvascular endothelium, and that differences in shear rates cannot explain the greater propensity for leukocyte rolling and adhesion in venules than arterioles. (J. Clin. Invest. 1991.87:1798-1804.) Key words: leukocyte adherence $\bullet$ inflammation • microvascular endothelium • neutrophils
\end{abstract}

\section{Introduction}

Blood flow-induced shear forces play an important role in disrupting the adhesive bonds between leukocytes and endothelium. The nature of this adhesive interaction has been studied both in vivo (1-3) and in vitro (4-6). In vitro studies indicate that there is little adherence of leukocytes to cultured endothelial cells at shear stresses above $2.0 \mathrm{dyn} \cdot \mathrm{cm}^{-2}(5,6)$, and the leukocyte adherence is independent of the CD11/CD18 adher-

Address correspondence and reprint requests to Dr. D. Neil Granger, Department of Physiology and Biophysics, Louisiana State University Medical Center, P.O. Box 33932, Shreveport, LA 71130-3932.

Received for publication 10 May 1990 and in revised form 4 January 1991.

J. Clin. Invest.

(c) The American Society for Clinical Investigation, Inc.

0021-9738/91/05/1798/07 \$2.00

Volume 87, May 1991, 1798-1804 ence glycoproteins except at shear stresses below $0.5 \mathrm{dyn} \cdot \mathrm{cm}^{-2}$ (6). However, the shear stress imposed in most in vitro models of leukocyte adhesion is much less than those encountered in vivo where values in $30-40-\mu \mathrm{m}$ diameter venules in the cat mesentery range from 3 to $36 \mathrm{dyn} \cdot \mathrm{cm}^{-2}$ with a mean of 17.5 dyn $\cdot \mathrm{cm}^{-2}(3)$.

In vivo studies indicate that the hydrodynamic force due to blood flow velocity and shear rate exerts considerable influence on leukocyte-endothelial cell interaction, however the importance of these forces in the absence of any proinflammatory stimulus is not clearly defined. In addition, the role of leukocyte adhesion molecules in this shear rate induced alteration in leukocyte-endothelial cell interaction has not been assessed in vivo. Thus the first objective of this study was to systematically analyze leukocyte-endothelial interactions in individual mesenteric venules over a wide range of shear rates and to assess the role of CD11/CD18 adhesion molecules on this interaction. Rather than relying on spontaneous changes in shear rate or compression of individual microvessels, we cannulated the arterial supply to the mesentery so that red cell velocity and shear rate could be varied over a wide range without direct interference to the vessel under observation.

An interesting and consistent observation is that leukocyteendothelial interactions in vivo are confined to the venular segment of the microcirculation and rarely observed in arterioles (1). Two explanations can be provided for the preferential adhesion of leukocytes to venular endothelium: the existence of lower shear rates in venules compared to arterioles (1), or a heterogeneous distribution of receptors for adhesion molecules between arterioles and venules (7).

As early as 1973, Atherton and Born (1) suggested that the reason leukocyte adhesion and rolling was not observed in arterioles was because the higher shear force exceeded the adhesive force in these vessels. One might predict, therefore, that reducing arteriolar shear rate may promote leukocyte adhesion in arterioles. The second explanation for the lack of adherence observed in arterioles is that the receptors for adhesive glycoproteins are preferentially distributed on the venular endothelium. Although this has been suggested (7) such a heterogeneous distribution of receptors is yet to be established. Therefore, the second objective of this study was to characterize leukocyte-endothelial interactions in arterioles over the same range of shear rates encountered in venules and to determine what role, if any, the CD11/CD18 adhesive glycoprotein complex plays in this interaction.

\section{Methods}

17 cats (1.4-3.5 kg) were fasted for 18-24 h and initially anesthetized with ketamine hydrochloride $(50 \mathrm{mg} / \mathrm{kg}$ i.m.). The jugular vein was cannulated, a saline drip established, and anesthesia was maintained with intravenous sodium pentobarbitone $(30 \mathrm{mg} / \mathrm{kg})$. A tracheotomy was performed and the animal ventilated by a respirator (model 665 , Harvard Apparatus Co. Inc., S. Natick, MA). Systemic arterial pressure 
was monitored by a Statham P23 pressure transducer connected to a catheter in the right carotid artery.

A midline abdominal incision was made and the proximal part of the small intestine (from close to the pylorus to the ligament of Trietz) and the large bowel (from the ileocecal valve to the distal colon) were surgically removed. The remaining isolated segment of small bowel was left undisturbed with blood and lymph vessels intact.

The animal was heparinized $(1,000 \mathrm{IU} / \mathrm{kg})$ and an arterial circuit established between the superior mesenteric artery (SMA) ${ }^{1}$ and the femoral artery. SMA blood flow was continuously monitored using an electromagnetic flowmeter (Carolina Medical Electronics, King, NC) connected to a probe positioned within the arterial circuit. SMA blood pressure was measured via a sideport in the flow probe. Systemic and SMA blood pressures were recorded with a Grass recorder (Grass Instruments Co., Quincy, MA). Body temperature was maintained at $37^{\circ} \mathrm{C}$ by a thermistor-controlled heat lamp. All exposed tissue was covered with saline-soaked gauze to avoid evaporation.

After completing surgery, the animals were placed in a left lateral recumbent position on an adjustable plexiglass microscope stage. A segment of midjejunum was exteriorized through the abdominal incision with great care taken to avoid trauma to the exposed bowel and mesentery. The mesentery was then prepared for in vivo microscopic observation according to the methods of House \& Lipowsky (3). The mesentery was draped over an optically clear viewing pedestal that allowed for transillumination of a $3-\mathrm{cm}^{2}$ section of mesentery. The pedestal was maintained at $37^{\circ} \mathrm{C}$ with a constant temperature circulator (model 80; Fisher Scientific Co., Pittsburgh, PA). The exposed bowel wall was draped with saline-soaked gauze, while the remainder of the mesentery was covered with Saran Wrap (Dow Corning Corp., Midland, MI). The exposed mesentery was suffused with a warmed $\left(37^{\circ} \mathrm{C}\right)$ bicarbonate-buffered salt solution $(\mathrm{pH} 7.4)$ at a rate of $2.0 \mathrm{ml} /$ $\min$. The oxygen tension of the suffusion solution was reduced to 40 $\mathrm{mmHg}$ by bubbling with a mixture of $5 \% \mathrm{CO}_{2}-95 \% \mathrm{~N}_{2}$.

The mesenteric microcirculation was observed through an intravital video microscope (Ortholux II E. Leitz, Inc., Rockleigh, NJ) with a $\times 20$ objective lens (Zeiss UD 20/0.60; Carl Zeiss, Inc., Thornwood, $\mathrm{NY}$ ) and a $\times 10$ eye piece. The mesentery was transilluminated with a 12 V-100 W DC-stabilized light source. A video camera (Dage, MTI) mounted on the microscope projected the image onto a black and white monitor (Cohu Inc., San Diego, CA), and the images were recorded using a video cassette recorder (Panasonic NV 8950). A video time-date generator (Panasonic WJ 810) projected the time, date, and stopwatch function onto the monitor. The final magnification was 1,400 .

Single unbranched arterioles and venules with diameters ranging between 25 and $35 \mu \mathrm{m}$ and $\sim 250 \mu \mathrm{m}$ in length were selected for study. Vessel diameter was measured with a video caliper (Microcirculation Research Institute, Texas A \& M University, College Station, TX). The number of adherent leukocytes was determined off-line during playback of videotaped images. An adherent leukocyte was defined as a leukocyte that adhered to the endothelium for a period equal to or greater than $30 \mathrm{~s}$ without rolling. Adherent cells were expressed as the number per $100 \mu \mathrm{m}$ length of venule. Red cell velocity $\left(V_{\text {roc }}\right)$ was measured using an optical Doppler velocimeter (Microcirculation Research Institute, Texas A \& M University, College Station, TX) that was calibrated against a rotating glass disk coated with red blood cells. Leukocyte rolling velocity $\left(V_{\text {wbc }}\right)$ was calculated from the time required for a leukocyte to traverse $50 \mu \mathrm{m}$ length of microvessel. In venules, a mean of 10 estimates of transit time was used to determine $V_{\text {wbc }}$ at each shear rate. Because of the lower incidence of leukocyte rolling in arterioles a mean of 8 estimates of transit time was used to estimate $V_{\text {wbc }}$. Rolling leukocytes were defined as those white blood cells that continued to move but at a velocity less than that of the erythrocytes and were

1. Abbreviations used in this paper: SMA, superior mesenteric artery; $V_{\text {rbc }}$, red cell velocity; $V_{\text {wbc }}$, leukocyte rolling velocity. in contact with the endothelial cell surface. Leukocytes that were adherent for 1-29 s were not considered as rollers.

Experimental protocol. Each vessel was videotaped for 2 min under baseline conditions, then the velocity of blood flow was reduced by tightening an adjustable screw clamp on the arterial circuit and a further 2-min recording made at reduced velocity. The clamp was then released and normal flow resumed for a period of $5 \mathrm{~min}$ to allow leukocyte adherence and rolling velocity to return to baseline values between each measurement. Velocity was reduced to either $75,50,25$, or $10 \%$ of the resting value in random order. Generally, no more than three velocity reductions were made in any one vessel because of the tendency for the baseline level of adherence to increase, particularly after severe flow reduction.

In 5 of the 17 animals the monoclonal antibody $(\mathrm{mAb}) \mathrm{IB}_{4}$ (directed against the leukocyte adhesion molecule CD18) was administered (1 $\mathrm{mg} / \mathrm{kg}$ i.v. [8]) and after $20 \mathrm{~min}$ the above recordings were repeated. Because the antibody blocked the tendency for baseline leukocyte adhesion to increase, approximately six velocity reductions could be used in each vessel.

Calculations. The leukocyte rolling velocity was expressed as a fraction of the red cell velocity $\left(V_{\text {wbc }} / V_{\text {rbc }}\right)$. Venular blood flow was calculated as the product of mean red cell velocity $\left[V_{\text {mean }}=\right.$ centerline velocity $\div 1.6(2)]$ and microvascular cross-sectional area assuming cylindrical geometry. Wall shear rate $(\gamma)$ was calculated using the Poiseuille's Law for a Newtonian fluid $\gamma=\left(V_{\text {mean }} / D\right) 8$, where $D$ is vessel diameter (9). Wall shear stress was calculated as $\gamma \times$ blood viscosity $(\eta)$ and $\eta$ was assumed to be 0.025 poise (10).

The data were analyzed using standard statistical analyses, i.e., oneway analysis of variance, and Student's $t$ test with a Bonferroni correction for multiple comparisons where necessary. All values are given as mean $\pm \mathrm{SE}$, and statistical significance was set at $P<0.05$.

\section{Results}

The protocol for an individual experiment is shown in Fig. 1. Local pressure in the arterial loop supplying the intestine and mesentery was reduced for periods of 2 min to $\sim 20$ and 10 $\mathrm{mmHg}$ in order to reduce $V_{\mathrm{rbc}}$ from a control value of $6 \mathrm{~mm} / \mathrm{s}$ to $3(50 \%)$ and $1.5(25 \%) \mathrm{mm} / \mathrm{s}$. Between each pressure reduction the clamp was released and local pressure returned to control. A reduction in $V_{\text {rbc }}$ was associated with a reduction in $V_{\text {wbc }}$ and an increased number of adherent leukocytes. These effects were largely reversed when full perfusion pressure was returned.

The relationship between leukocyte rolling velocity and red cell velocity when red cell velocity was varied by reducing local perfusion pressure is shown in Fig. 2. There was a linear decline in the velocity of white cells as red cell velocity was reduced from 6,000 to $500 \mu / \mathrm{s}$. Treatment with the CD18-specific antibody $\mathrm{mAb} \mathrm{IB}_{4}$ did not alter the slope of the relationship but caused a significant $(P<0.01)$ increase in the intercept from 9.6 to 28.0 . That is, $\mathrm{mAb} \mathrm{IB}_{4}$ appears to increase leukocyte rolling velocity, however, this effect is only apparent at red cell velocities below $2,000 \mu \mathrm{m} / \mathrm{s}$.

The ratio of leukocyte rolling velocity to red cell velocity $\left(V_{\text {wbc }} / V_{\text {rbc }}\right)$ at different shear rates is shown in Fig. 3. In untreated animals, the ratio is relatively constant at 0.025 over the entire range of shear rates studied. Immunoneutralization of leukocyte adhesion molecules with $\mathrm{mAb} \mathrm{IB}_{4}$ resulted in an increased leukocyte rolling velocity and caused the ratio $V_{\text {wbc }} / V_{\text {rbc }}$ to increase, particularly at low shear rates.

The number of adherent leukocytes in each venule also increased as red cell velocity and wall shear rate were reduced (Fig. 4). At shear rates below $250 \mathrm{~s}^{-1}$ there was a mean of $6 \pm 1$ leukocytes adherent per $100 \mu \mathrm{m}$ length of venule. 


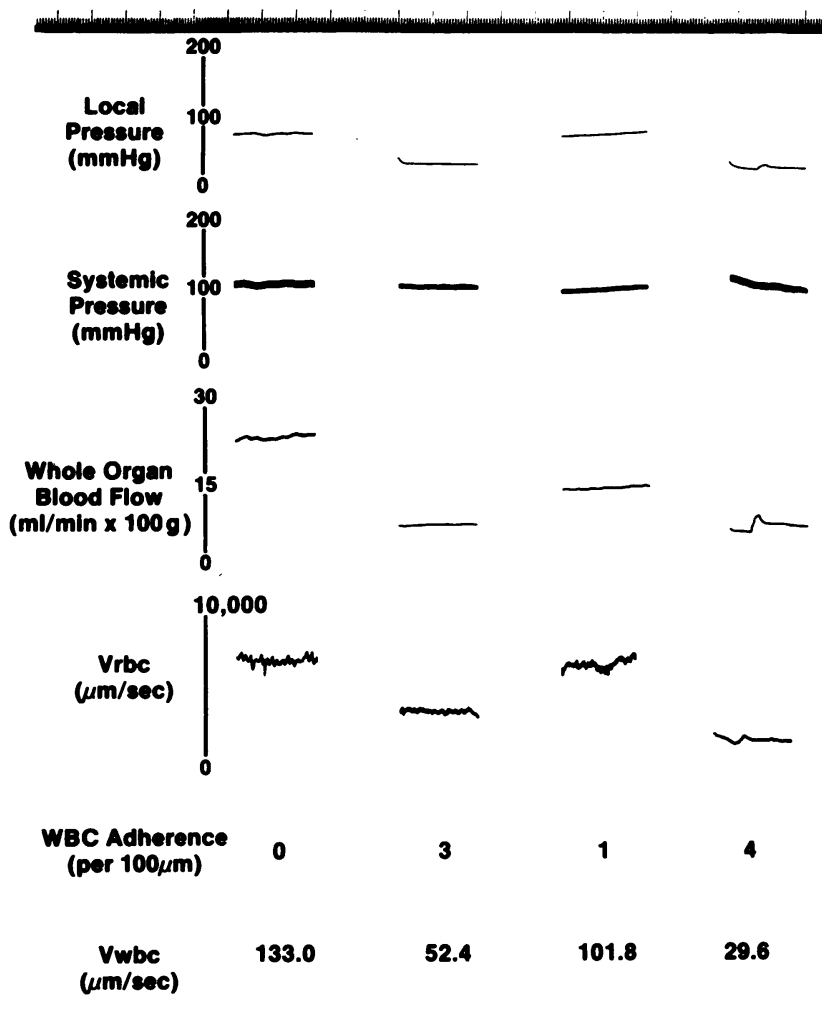

Figure 1. Results of an individual experiment in which a reduction in local perfusion pressure was used to reduce $V_{\mathrm{rbc}}$ in a mesenteric venule in the cat. The reduction in $V_{\mathrm{rtb}}$ was associated with a fall in the $V_{\text {wbc }}$ and an increase in leukocyte adherence. These effects were largely reversible when local pressure was returned to control.

An even closer relationship was observed between the number of adherent leukocytes and leukocyte rolling velocity (Fig. $5)$, such that when $V_{\text {wbc }}$ fell below $50 \mu \mathrm{m} / \mathrm{s}$ there was a progressive increase in adherence. The increase in adherence observed at low shear rate or low leukocyte rolling velocity was largely abolished by administration of $\mathrm{mAb} \mathrm{IB}_{4}$ (Figs. 4 and 5).

In order to test that the effects of $\mathrm{mAb} \mathrm{IB}_{4}$ were specific for CD11/CD18, animals were given $\mathrm{R} 3.1(1 \mathrm{mg} / \mathrm{kg})$, an antibody that does not bind to CD11/CD18 on feline leukocytes (as as-

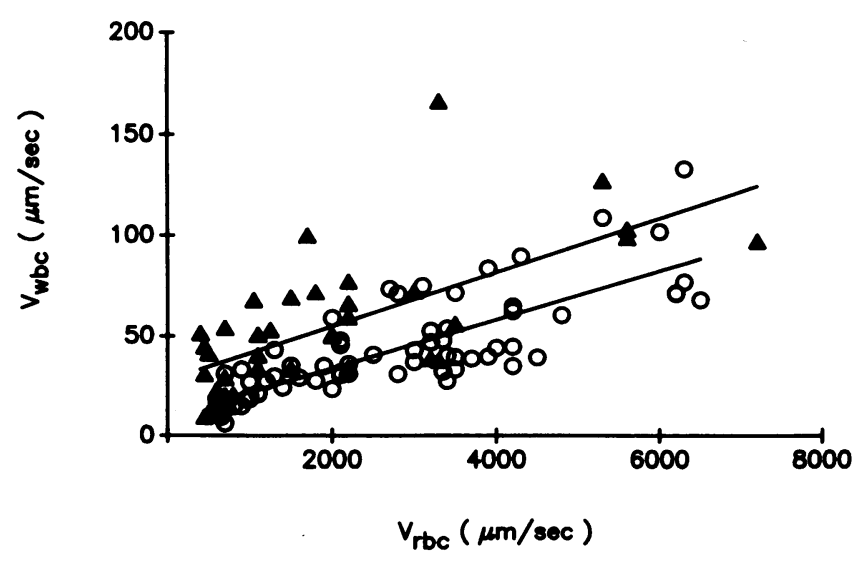

Figure 2. The effect of reducing $V_{\mathrm{rbc}}$ on $V_{\text {wbc }}$ in cat mesenteric venules. In untreated animals $(0)$ the relationship is: $Y=12.1 \times+9.6(r$ $=0.67)$, and in animals treated with $\mathrm{mAb} \mathrm{IB}_{4}(\Delta)$ is $Y=13.4 \times+28.0$ $(r=0.80)$.
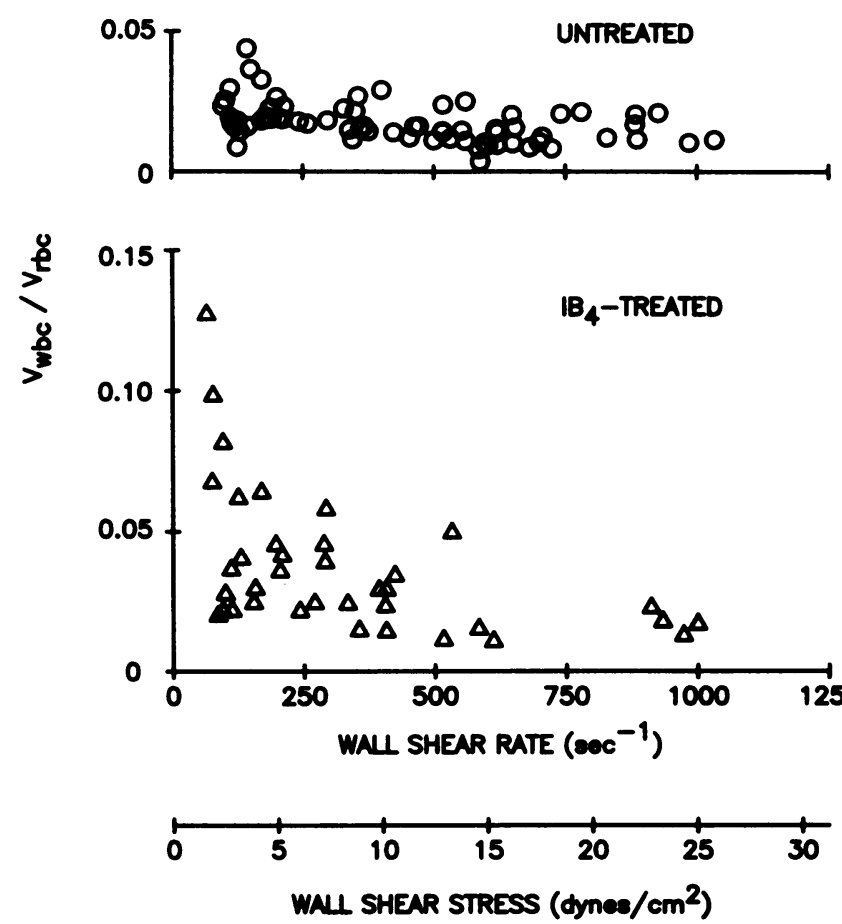

Figure 3 . The ratio of white cell velocity to red cell velocity ( $\left.V_{\text {wbc }} / V_{\text {rtc }}\right)$ expressed as a function of wall shear rate and wall shear stress in mesenteric venules of untreated animals and animals treated with the CD18-specific monoclonal antibody $\mathrm{IB}_{4}$.

sessed by flow cytometry). The results are shown in Fig. 6 . There was no difference between untreated animals and animals treated with R 3.1 in either $V_{\text {wbc }} / V_{\text {rbc }}$ or in WBC adherence.

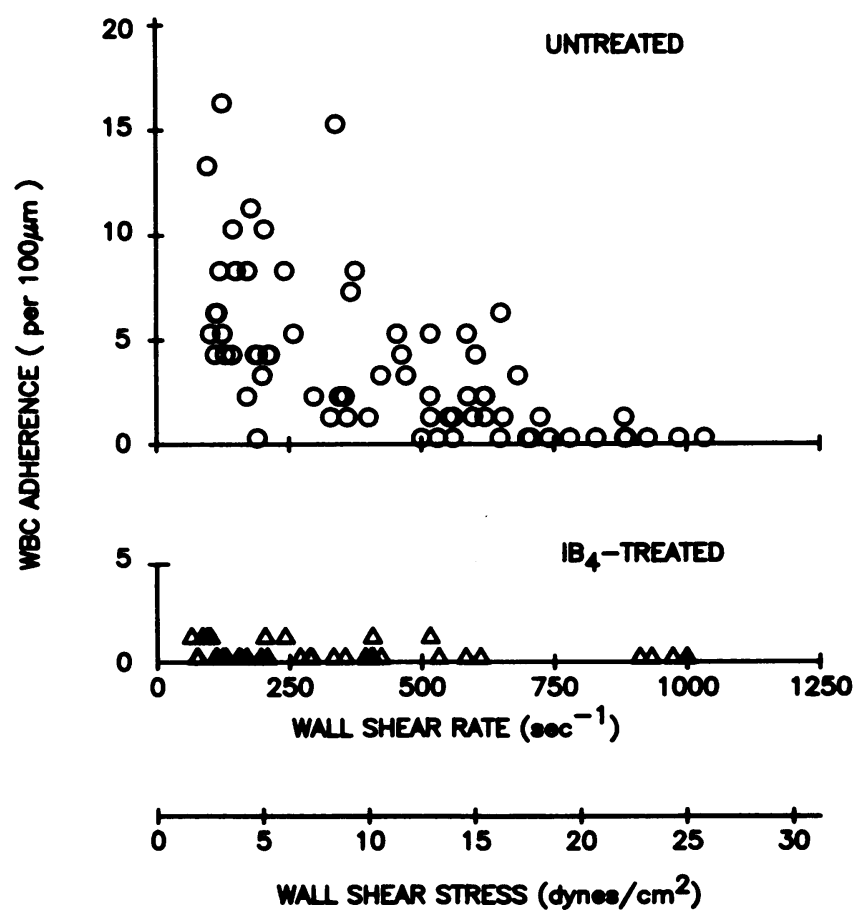

Figure 4. Leukocyte adherence in mesenteric venules plotted against wall shear stress. The increase in leukocyte adherence observed at shear stresses below 20 dynes $/ \mathrm{cm}^{2}$ is abolished by the monoclonal antibody $\mathrm{IB}_{4}$. 


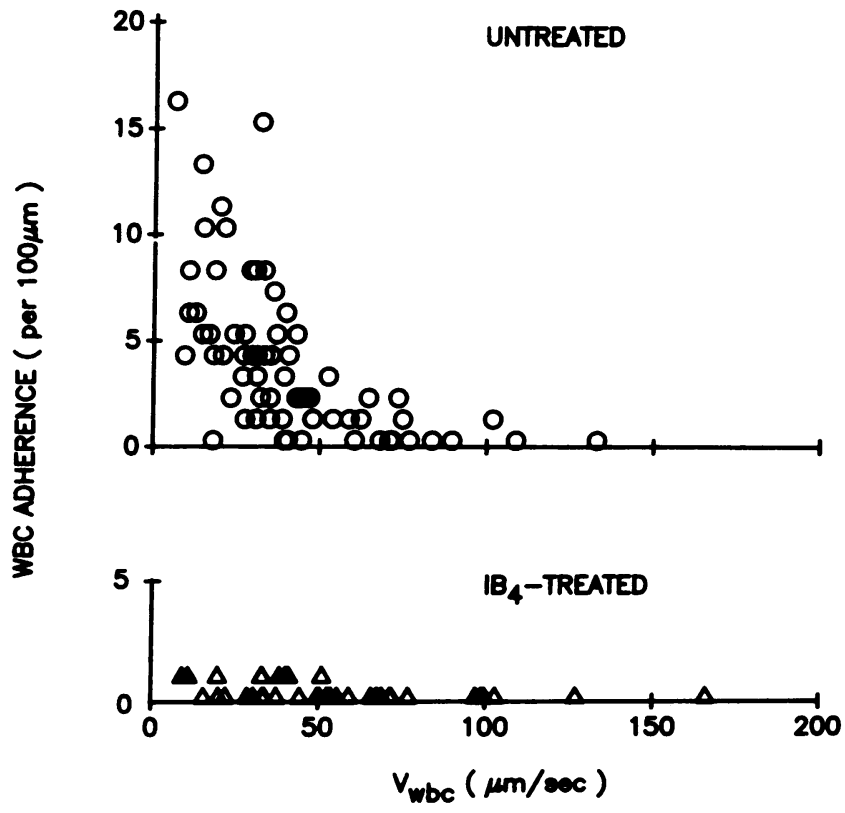

Figure 5. Leukocyte adherence in mesenteric venules plotted against $V_{\text {wbc }}$. Leukocyte adherence is particularly noticeable at $V_{\text {wbc }}$ below $50 \mu \mathrm{m} / \mathrm{s}$ and is abolished by the CD18-specific monoclonal antibody $\mathrm{IB}_{4}$.
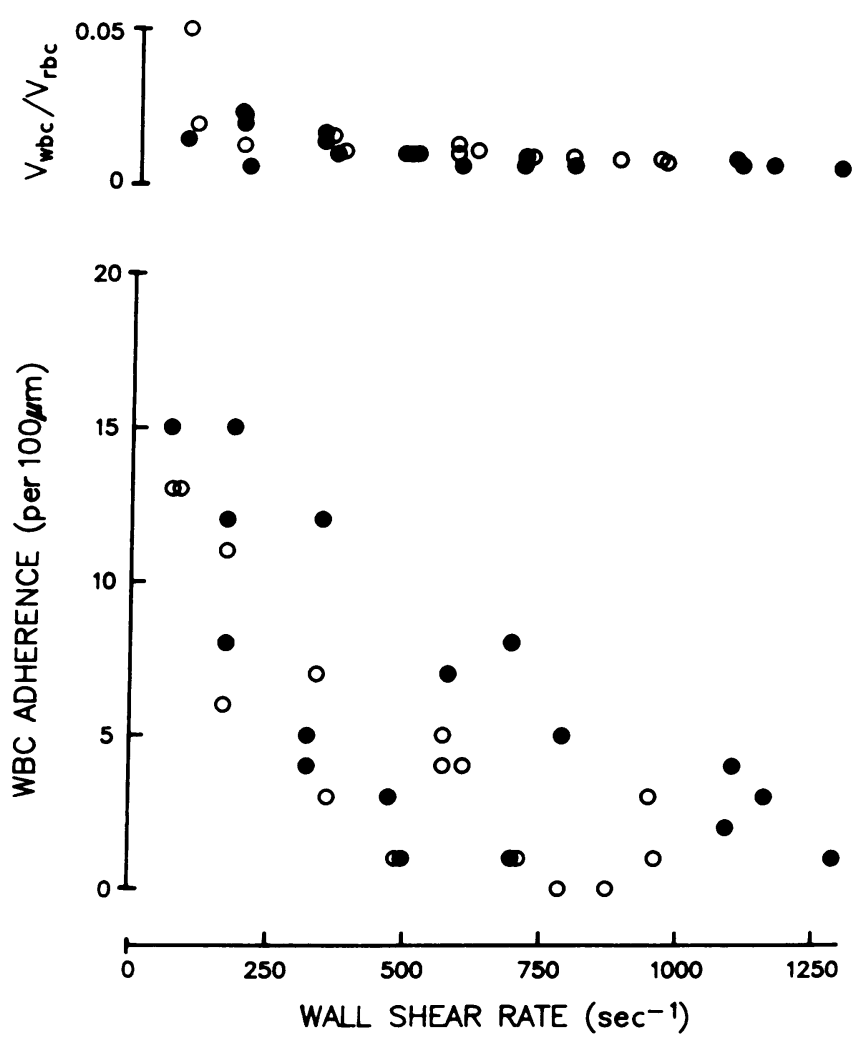

Figure 6 . The relationships among ratio of leukocyte rolling velocity to red blood cell velocity (above), white blood cell adherence (below), and wall shear rate in mesenteric venules of untreated animals (o) (from Figs. 3 and 4 ) and in animals treated with R 3.1 (๑), a monoclonal antibody which does not bind to CD11/CD18 on feline leukocytes.

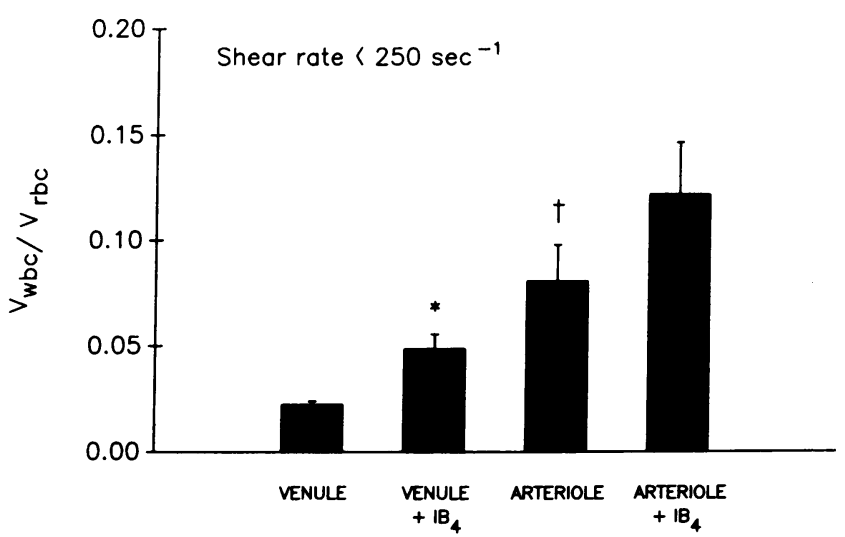

Figure 7. The ratio of $V_{\text {wbo }} / V_{\text {rbc }}$ in arterioles and venules in the cat mesentery at shear rates $<250 \mathrm{~s}^{-1}$. The monoclonal antibody $\mathrm{IB}_{4}$ significantly increased $V_{\text {wbc }} / V_{\text {rbc }}$ in venules but the effect was not significant in arterioles. ${ }^{*} P<0.05$ compared with untreated vessels. ${ }^{\dagger} P$ $<0.05$ compared with venules.

In addition to investigating the behaviour of leukocytes in venules, we studied their behavior in mesenteric arterioles. Leukocyte rolling and adherence only occurred in arterioles at low shear rates, generally $<250 \mathrm{~s}^{-1}$. At shear rates above 250 $\mathrm{s}^{-1}$ there was virtually no adherence and no obvious leukocyte rolling. Fig. 7 compares the ratio of leukocyte rolling velocity with red cell velocity in venules and arterioles at shear rates $<250 \mathrm{~s}^{-1}$ and in the presence or absence of $\mathrm{mAb} \mathrm{IB}_{4}$. The mean shear rate in each group was $158 \pm 9(n=23)$ for venules, $133 \pm 12(n=18)$ for venules plus $\mathrm{mAb} \mathrm{IB}_{4}, 115 \pm 26(n=6)$ for arterioles, and $85 \pm 8(n=4)$ for arterioles plus $\mathrm{mAb} \mathrm{IB}_{4}$. The mean value of $V_{\text {wbd }} / V_{\text {rbc }}$ for venules was 0.02 . The addition of $\mathrm{mAb} \mathrm{IB}_{4}$ increased leukocyte rolling velocity and doubled the ratio $V_{\text {wbc }} / V_{\text {rbc }}$. In arterioles, the leukocytes rolled faster and the ratio $V_{\text {wbc }} / V_{\text {rbc }}$ was four times that observed in venules. This ratio tended to increase in arterioles after the addition of $\mathrm{mAb}$ $\mathrm{IB}_{4}$, however, this effect was not statistically significant.

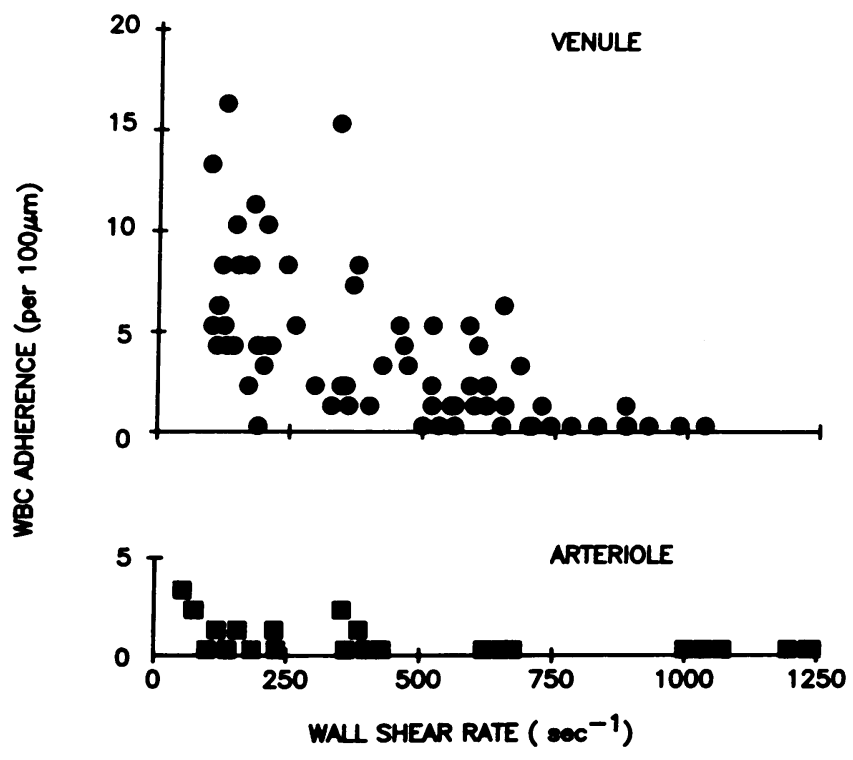

Figure 8. Leukocyte adherence expressed as a function of wall shear rate in arterioles and venules of the cat mesentery. 
Leukocyte adherence in both arterioles and venules is shown in Fig. 8 over a wide range of shear rates. Adherent leukocytes were commonly observed in venules up to shear rates of $750 \mathrm{~s}^{-1}$. However, in arterioles adherence was only observed at shear rates $<385 \mathrm{~s}^{-1}$ and rarely rose above one adherent leukocyte per $100 \mu \mathrm{m}$ length of vessel. In the presence of $\mathrm{mAb} \mathrm{IB}_{4}$ no adherent leukocytes were observed in any of the arterioles studied.

\section{Discussion}

Our data indicate that as red cell velocity falls there is a linear decline in the rolling velocity of white cells, with a tendency for white cell velocity to fall more rapidly at red cell velocities below $1.0 \mathrm{~mm} / \mathrm{s}$. Atherton and Born (1) reported similar findings but with a definite reduction in slope of the relationship at $V_{\text {rbc }}$ above $1.0 \mathrm{~mm} / \mathrm{s}$, while Firrell \& Lipowsky (2) found that $V_{\text {wbc }}$ remained constant once $V_{\text {rbc }}$ rose above $1 \mathrm{~mm} / \mathrm{s}$. In this study individual venules of similar diameter were chosen and $V_{\text {rbc }}$ altered over a wide range. Atherton \& Born relied on the spontaneous velocities observed in vessels of markedly different sizes (30-200 $\mu \mathrm{m})$, while Firrell and Lipowsky combined spontaneously observed velocities in different sized vessels with compression of some vessels in order to accumulate observations over a wide range of velocities. Because $V_{\mathrm{rbc}}$ increases as vessel size increases, it is likely that the higher $V_{\text {rbc }}$ values represent data accumulated in larger venules, so that the plateau in the relationship between $V_{\text {wbc }}$ and $V_{\text {rbc }}$ observed by these two groups may reflect an effect of vessel size on $V_{\text {wbc }}$.

Although our data strongly suggest that $V_{\text {wbc }}$ increases with increase in $V_{\mathrm{rbc}}$ we have only investigated reductions in flow. We cannot exclude the possibility that at higher than normal velocities there is a plateau in this relationship.

Another index used to assess leukocyte-endothelial interaction is the ratio $V_{\mathrm{wbc}} / V_{\mathrm{rbc}}$. This ratio provides a measure of the fracture stress at the area of contact between leukocytes and endothelium. The higher the velocity ratio the lower the amount of energy required to peel a leukocyte from the microvessel wall (11). This ratio has been used to assess the effect of various agents on leukocyte rolling, e.g., we have shown that superoxide dismutase given $1 \mathrm{~h}$ after ischemia caused a significant increase in $V_{\text {wbc }} / V_{\text {rbc }}$ without altering $V_{\text {rbc }}(8)$. Agents that alter $V_{\text {wbc }} / V_{\mathrm{rbc}}$ and at the same time alter $V_{\mathrm{rbc}}$ make interpretation of data difficult because of the uncertainty regarding the influence of hydrodynamic dispersal forces on this ratio. This study is the first to systematically assess the effects of shear force on this ratio by altering shear rate in individual venules. The results indicate that in untreated animals the ratio remains constant over a wide range of shear rates. That is, $V_{\text {wbc }} / V_{\text {rbc }}$ is a useful tool to assess leukocyte-endothelial interactions even in situation where $V_{\text {rbc }}$ changes.

\section{Factors affecting leukocyte adherence}

Although leukocyte rolling is commonly observed in venules, the adherence of leukocytes to venular endothelium is rare in the absence of a proinflammatory stimulus. Physiologic factors that modify leukocyte adhesion include $(a)$ adhesion molecules expressed on the surface of the activated leukocyte and/or endothelial cell $(12,13),(b)$ electrostatic charge interaction between leukocyte and endothelial cell surfaces $(14,15)$ including effects due to the presence of sialic acid residues (16), and (c) hydrodynamic dispersal forces such as wall shear stress that tend to sweep leukocytes away from the microvessel wall $(1,3)$.
Shear forces. In vitro studies indicate that shear stress plays a major role in determining the extent of leukocyte adherence to endothelium. Lawrence et al. (4) found that FMLP-stimulated neutrophil adherence to cultured human umbilical vein endothelial cells was completely abolished by a shear stress of $3.9 \mathrm{dyn} / \mathrm{cm}^{2}$. Other studies indicate that the critical shear stress required to overcome adhesion may be as low as $1-2 \mathrm{dyn} / \mathrm{cm}^{2}$ $(5,6)$. These findings contrast with the present in vivo study in which a shear stress of $\sim 18 \mathrm{dyn} / \mathrm{cm}^{2}$ (shear rate of $750 \mathrm{~s}^{-1}$ ) was required to abolish adherence. As shear stress was reduced from 18 down to $6 \mathrm{dyn} / \mathrm{cm}^{2}$ adherence increased progressively, and below $6 \mathrm{dyn} / \mathrm{cm}^{2}$ adherence increased dramatically. Thus, our data indicate that the adhesive forces in vivo are far greater than the dispersive forces at shear stresses below $6 \mathrm{dyn} / \mathrm{cm}^{2}$, while the adhesive forces are clearly weaker and rarely exceed $2-3 \mathrm{dyn} / \mathrm{cm}^{2}$ in various in vitro models. Schmid-Schoenbein et al. (17) calculated the shear stress associated with a leukocyte adhering to venular endothelium in the rabbit omentum and found a range of $50-1,060 \mathrm{dyn} / \mathrm{cm}^{2}$. The lower shear stress values observed in vitro may reflect a lower expression of adhesion receptors on cultured endothelium compared with vessels in vivo, however this remains to be investigated.

Leukocyte rolling velocity. Before a leukocyte can adhere to the wall of a blood vessel it must first roll along the surface of endothelial cells. Although it is generally accepted that leukocyte rolling precedes adherence, there is no quantitative assessment in the literature of the correlation between leukocyte rolling velocity and leukocyte adherence. Our results indicate that leukocyte adherence is minimal at leukocyte rolling velocities above $50 \mu \mathrm{m} / \mathrm{s}$, however, once the velocity falls below 50 $\mu \mathrm{m} / \mathrm{s}$, adherence increases dramatically and reaches a maximum of 10-16 adherent leukocytes per $100 \mu \mathrm{m}$ length of vessel at rolling velocities of $5-20 \mu \mathrm{m} / \mathrm{s}$. This increase in adherence may result from the fact that a progressive decrease in rolling velocity would lead to a progressive increase in contact time between adhesive molecules expressed on the surface of leukocytes and/or endothelium. Once a critical contact time is achieved the adhesive interaction between leukocytes and endothelium is sufficiently strong to permit adherence.

Leukocyte adhesion molecules. Another important determinant of whether leukocytes adhere to endothelium at any given shear rate is the proadhesive force generated by leukocyte adhesion molecules. The leukocyte adhesion glycoprotein complex termed CD11/CD18 (or MAC-1; LFA-1; p150,95) is the primary mediator of adhesion of activated leukocytes to endothelial cells $(12,13,15)$. These are three structurally and functionally related glycoprotein heterodimers each consisting of an immunologically distinct $\alpha$ subunit (CD11) that is noncovalently associated with a common $\beta$ subunit (CD18). These adhesive molecules are rapidly mobilized in response to various proinflammatory mediators $(18,19)$. In normal, nonactivated neutrophils there is low level expression of CD11/CD18 on leukocytes. When the neutrophil is exposed to a proinflammatory stimulus there is rapid upregulation of a specific subunit (CD1 1b/CD18) (13). Our study indicates that the CD11/CD18 complex plays a major role in the shear rate-dependent leukocyte adhesive interactions observed in postcapillary venules. Immunoneutralization of the common $\beta$ chain of CD11/CD18 with monoclonal antibody $\mathrm{IB}_{4}$ significantly improved leukocyte rolling velocity at any given red cell velocity and abolished the leukocyte adherence normally observed at low shear rates.

The energy associated with the attachment of a leukocyte to 
the endothelium is termed adhesion energy. A measure of this energy and therefore the strength of the intercellular bond is the fracture stress. Schmid-Schonbein and co-workers (11) have shown that the fracture stress is a function of the ratio $V_{\text {wbc }} / V_{\text {rbc }}$ such that as this ratio decreases fracture stress increases, indicating an increase in adhesion energy. In this study immunoneutralization of CD11/CD18 dramatically increased $V_{\text {wbc }} / V_{\text {rbc }}$ at shear rates $<250 \mathrm{~s}^{-1}$, indicating that there was a dramatic reduction in adhesion energy in the presence of $\mathrm{IB}_{4}$.

Our observations that wall shear stresses below 15 dynes/ $\mathrm{cm}^{2}$ (the normal range of shear stress encountered in vivo in the cat mesentery is 3-36 dynes/ $\mathrm{cm}^{2}$ ) result in CD11/CD18-dependent leukocyte adhesion is inconsistent with several in vitro studies which indicate that activated leukocytes are unable to adhere to endothelial cell monolayers at shear stresses $>2-3$ dynes $/ \mathrm{cm}^{2}$ (4-6). One explanation for this difference in adherence at wall shear stresses above 3 dynes $/ \mathrm{cm}^{2}$ is that the medium used in vitro does not mimic the conditions in vivo in which leukocytes are suspended in a complex solution containing various proteins and other cell types. The inability of the leukocytes to form strong adhesive interactions in vitro that can withstand high shear forces may also be the result of low level expression of the ligand for CD11/CD18 on the surface of the cultured endothelial cells. Because leukocytes have to be isolated from whole blood for in vitro studies, the process of isolation may interfere with or reduce the expression of CD11/CD18 glycoproteins relative to the same cells in vivo. The nature of the endothelium may be an important determinant of adhesive interaction. Most in vitro studies employ cultured endothelial cells from either human umbilical vein or bovine pulmonary artery. Leukocytes are far less likely to develop adhesive interactions with major vessels, particularly those on the arterial side of the circulation, than with venules or capillaries. It is possible that endothelial cells cultivated from venules may show a stronger adhesive interaction with leukocytes in vitro than cells taken from arteries or veins. Finally, one cannot exclude the possibility that there are variations in the strength of adhesive interaction in different species, however this remains to be established.

The values for wall shear stress in this study are calculated by assuming a value for blood viscosity of 0.025 poise. This value was derived in cat mesenteric venules of $30 \mu \mathrm{m}$ diameter (10). It is possible that the effective viscosity within the microcirculation may be lower than 0.025 poise in which case the shear stress would be reduced. Even if the viscosity was assumed to be as low as that of plasma ( 0.01 poise) this data would still indicate that the adherence of leukocytes in vivo is greater than that observed in vitro for a given shear stress.

\section{Adherence at low shear rates}

An important observation of this study was the progressive increase in the number of adherent leukocytes when wall shear rate was reduced below $750 \mathrm{~s}^{-1}$. The mechanisms underlying leukocyte adhesion at low wall shear rates are not clear. One explanation for this behaviour is that there is a low level of CD11/CD18 expression in normal circulating leukocytes. When shear rate exceeds $750 \mathrm{~s}^{-1}$ the proadhesive force generated by these adhesion molecules is not sufficient to overcome the anti-adhesive force generated by shear, however, as shear rate is reduced the balance between proadhesive and hydrodynamic dispersal forces changes such that leukocytes adhere.
Thus, while a low level expression of CD18 may result in minimal adhesion at normal shear stresses, a strong adhesive interaction may develop between leukocytes and endothelium at low shear rates because contact time is increased allowing greater opportunity for adhesive bonds to be formed between leukocyte and endothelial cell. An alternative explanation for the increased adherence at low shear rate is that there is minimal or no expression of CD11/CD18 on circulating leukocytes, but as venular blood flow is reduced there is a concomitant reduction in the washout of endogenous proinflammatory agents normally produced by endothelial cells. At shear rates $<750 \mathrm{~s}^{-1}$ this accumulation of inflammatory agents would lead to expression of CD11/CD18 on rolling leukocytes and ultimately to adherence. The lower the shear rate the greater the adherence. In this scheme immunoneutralization of CD18 with $\mathrm{IB}_{4}$ should prevent the rise in leukocyte adherence at low shear rates.

\section{Adherence: arterioles versus venules}

A consistent observation in the literature is that leukocyte rolling and adhesion is much less common in arterioles than in venules. One possible explanation for this decreased adherence is the greater shear rate in arterioles compared with venules. If shear rate was a major deterrent to leukocyte adherence in arterioles then reducing shear rate in these vessels should lead to leukocyte rolling and adhesion. The results of our study indicate that shear forces do contribute to the absence of leukocyte rolling and adhesion, i.e., rolling and adherence was observed in arterioles exposed to low $\left(<250 \mathrm{~s}^{-1}\right)$ shear rates. However, the magnitude of leukocyte adhesion as well as the velocity of leukocyte rolling is dramatically different between arterioles and venules exposed to the same shear rate. In arterioles, adherence was never observed at shear rates above $385 \mathrm{~s}^{-1}$, while at shear rates $<250 \mathrm{~s}^{-1}$ adherence rarely rose above 2 per $100 \mu \mathrm{m}$, while in venules adherence was observed at shear rates up to $880 \mathrm{~s}^{-1}$, and rose as high as 12-16 adherent leukocytes per $100 \mu \mathrm{m}$ at shear rates $<250 \mathrm{~s}^{-1}$ (Fig. 7). Similarly, the ratio of leukocyte rolling velocity to red cell velocity was four times greater in arterioles than venules at shear rates $<250 \mathrm{~s}^{-1}$. These results indicate that at any given shear rate the level of adhesive interaction between leukocytes and endothelial cells is much less in arterioles than in venules.

The greater interaction of leukocytes with venular endothelium could be due, in part, to the hydrodynamic interaction of leukocytes with red blood cells which results in the leukocytes being preferentially displaced towards the wall of the vessel (20). This phenomenon is thought to occur as vessel diameter increases at the level of postcapillary venules and may enhance the likelihood of leukocyte rolling and adhesion.

The low level of adherence observed in arterioles at low shear rates does appear to be CD18 dependent. After administration of monoclonal antibody $\mathrm{IB}_{4}$ to the circulation we never observed an adherent leukocyte in any arteriole. However, the role of CD11/CD18 in leukocyte rolling in arterioles is uncertain. In this study the tendency for $V_{\text {wbc }} / V_{\text {rbc }}$ to increase in the presence of $\mathrm{IB}_{4}$ was not significant.

Although CD18 plays a role in leukocyte adherence in arterioles at low shear rates, the level of adhesion in arterioles is low and this may reflect a lower density of the ligand for CD11/CD18 on arteriolar endothelium compared with venular endothelium. Alternatively, the arteriole may normally produce an anti-adhesive factor that is not produced by venules. 


\section{Acknowledgments}

The authors thank Janice Russell for her invaluable technical assistance in all aspects of this study. The authors also thank Dr. Karl Arfors and Dr. Robert Rothein for providing monoclonal antibodies $\mathrm{IB}_{4}$ and R3.1, respectively.

This work was supported by a grant from the National Institutes of Health (DK-35594).

\section{References}

1. Atherton, A., and G. V. R. Born. 1973. Relationship between the velocity of rolling granulocytes and that of the blood flow in venules. J. Physiol. 233:157165.

2. Firrell, J. C., and H. H. Lipowsky. 1989. Leukocyte margination and deformation in mesenteric venules of rat. Am. J. Physiol. 256:H1667-H1674.

3. House, S. D., and H. H. Lipowsky. 1987. Leukocyte-endothelium adhesion: microhemodynamics in mesentry of the cat. Microvasc. Res. 34:363-379.

4. Lawrence, M. B., L. V. McIntire, and S. G. Esken. 1987. Effect of flow on polymorphonuclear leukocyte endothelial cell adhesion. Blood. 70:1284-1290.

5. Lawrence, M. B., C. W. Smith, S. G. Eskin, and L. V. McIntire. 1990. Effect of venous shear stress on CD18-mediated neutrophil adhesion to cultured endothelium. Blood. 75:227-237.

6. Worthen, G. S., L. A. Smedly, M. G. Tonnesen, D. Ellis, N. F. Voelkel, J. J. Reeves, and P. M. Henson. 1987. Effects of shear stress on adhesive interaction between neutrophils and cultured endothelial cells. J. Appl. Physiol. 63:20312041.

7. Tsai, H. M., I. I. Sussman, R. L. Nagel, and D. K. Kaul. 1990. Desmopressin induces adhesion of normal human erythrocytes to the endothelial surface of a perfused microvascular preparation. Blood. 75:261-265.

8. Suzuki, M., W. Inauen, P. R. Kvietys, M. B. Grisham, C. Meininger, M. E. Schelling, H. J. Granger, and D. N. Granger. 1989. Superoxide mediates reperfusion-induced leukocyte-endothelial cell interactions. Am. J. Physiol. 257:H1740 H1745.
9. Lipowsky, H. H., S. Kovalcheck, and B. W. Zweifach. 1978. The distribution of blood rheological parameters in the microvasculature of cat mesentery. Circ. Res. 43:738-749.

10. Lipowsky, H. H., S. Usami, and S. Chien. 1980. In vivo measurements of "apparent viscosity" and microvessel hematocrit in the mesentry of the cat. $\mathrm{Mi}$ crovasc. Res. 19:297-319.

11. Schmid-Schonbein, G. W., R. Skalak, S. I. Simon, and R. L. Engler. 1987. The interaction between leukocytes and endothelium in vivo. Ann. NY Acad. Sci. 416:348-361.

12. Janoff, A., and B. W. Zweifach. 1964. Adhesion and emigration of leukocytes produced by cationic proteins and lysosomes. Science (Wash. DC). 144:1456-1458.

13. Tonnesen, M. G. 1989. Neutrophil-endothelial cell interactions: mechanisms of neutrophil adherence to vascular endothelium. J. Invest. Dermatol. 93:535-585.

14. Boxer, L. A., B. Bjorsten, J. Bjork, H. H. Lang, J. M. Allen, and R. L. Bachner. 1982. Neutropenia induced by systemic infusion of lactoferrin. J. Lab. Clin. Med. 99:866-772.

15. Rozen, H., and S. Gordon. 1989. Current status review: adhesion molecules and myelomonocytic cell-endothelial interactions. Br. J. Exp. Path. 70:385-394.

16. Görög, P., and G. V. R. Born. 1982. Increased adhesiveness of granulocytes in rabbit ear-chamber blood vessels perfused with neuraminidase. Microvasc. Res. 23:380-384.

17. Schmid-Schoenbein, G. W., Y.-C. Fung, and B. W. Zwerfach. 1975. Vascular endothelium-leukocyte interaction. Sticking shear force in venules. Circ. Res. 36:173-184.

18. Hoover, R. L., R. Folger, W. A. Haering, B. R. Ware, and M. J. Karnovsky. 1980. Adhesion of leukocytes to endothelium: roles of divalent cations, surface charge, chemotactic agents and substrate. J. Cell Sci. 45:73-82.

19. Zimmerman, G. A., and H. R. Hill. 1984. Inflammatory mediators stimulate granulocyte adherence to cultured human endothelial cells. Thromb. Res 35:203-217.

20. Schmid-Schönbein, G. W., S. Usami, R. Skalak, and S. Chien. 1980. The interaction of leukocytes and erythrocytes in capillary and postcapillary vessels. Microvasc. Res. 19:45-70. 